\title{
Histogram-based method for contrast measurement
}

\author{
Luis Miguel Sanchez-Brea, Juan Antonio Quiroga, Angel Garcia-Botella, and Eusebio Bernabeu
}

\begin{abstract}
A histogram-based technique for robust contrast measurement is proposed. The method is based on fitting the histogram of the measured image to the histogram of a model function, and it can be used for contrast determination in fringe patterns. Simulated and experimental results are presented. (C) 2000 Optical Society of America

OCIS codes: $100.0100,100.2650,100.2960$.
\end{abstract}

\section{Introduction}

Contrast measurement is a useful tool for the measurement of the modulation transfer function (MTF) of an optical system. A direct method for determining the MTF is by measuring the contrast of several sinusoidal fringe patterns with different spatial frequencies imaged by the optical system under test. However, since sinusoidal patterns are difficult to obtain, usually the MTF is indirectly measured with Ronchi patterns. This gives an estimation of the contrast transfer function (CTF). By means of a modal expansion of the square pattern, it is possible to obtain a relationship between the CTF and the MTF, and when this relationship is inverted, the MTF can be measured in terms of the $\mathrm{CTF}, 1,2$

$$
\begin{aligned}
\operatorname{MTF}(f)= & \frac{\pi}{4}\left[\operatorname{CTF}(f)+\frac{\operatorname{CTF}(3 f)}{3}-\frac{\operatorname{CTF}(5 f)}{5}\right. \\
& \left.+\frac{\operatorname{CTF}(7 f)}{7}+\cdots\right]
\end{aligned}
$$

The authors are with the Departamento de Optica, Universidad Complutense de Madrid, Facultad de Ciencias Físicas, Cuidad Universitaria s/n, 28040 Madrid, Spain. When this research was performed, J. A. Quiroga was with the Centro de Investigaciones en Óptica, Apartado Postal 1-948, 37000 Léon GTO, México. L. M. Sanchez-Brea's e-mail address is optbrea@eucmos.sim. ucm.es.

Received 13 October 1999; revised manuscript received 27 March 2000.

0003-6935/00/234098-09\$15.00/0

(C) 2000 Optical Society of America where $f$ is the frequency. In the absence of noise and for a periodic signal the straightforward definition of contrast is

$$
C=\frac{I_{\mathrm{MAX}}-I_{\mathrm{MIN}}}{I_{\mathrm{MAX}}+I_{\mathrm{MIN}}},
$$

where $I_{\mathrm{MAX}}$ and $I_{\mathrm{MIN}}$ are the maximum and the minimum values of the signal.

When noise or small background variations are present, it is not possible to apply this definition directly. If the image from which we wish to determine the contrast is formed by a straight-line pattern, one possibility is to align the pattern (by digital or optical methods) parallel to one of the reference axes and then sum along it. In this way the averaging will reduce the noise, enabling the measurement of $I_{\mathrm{MAX}}$ and $I_{\mathrm{MIN}}$. Unfortunately this simple method has big drawbacks, especially if mediumlow contrasts are to be measured $(C \approx 0.5-0.1)$. In addition, the determination of $I_{\mathrm{MAX}}$ and $I_{\mathrm{MIN}}$ by a simple averaging is sensitive to noise and/or to small background variations. Furthermore, this method cannot be applied when fringes become curved after passing through the optical system or for circular fringes, which can be used for the determination of directional MTF when we analyze a small slit of the image at the proper direction.

Another way to evaluate the contrast of a fringe pattern is by use of the histogram, as suggested by Lai and von Bally. ${ }^{3}$ However, the algorithm they propose does not match the definition of contrast [Eq. (2)] even when no noise is present. In this study we propose a histogram-based technique for contrast measurement of fringe patterns that can be applied in the presence of additive noise and with patterns composed of fringes that are not straight. The method is based on fitting the histogram of the measured fringe pattern to the histogram of a model func- 
tion that depends on several parameters. The parameters of the model function provide information about contrast and noise level.

By means of histogram analysis we can obtain more information about fringes, besides contrast. Generally histograms of fringe patterns present two lobes, but square and sinusoidal fringe patterns with the same contrast do not have the same histogram shape. From this difference, information about the fringe shape can be obtained.

This paper is organized as follows: In Section 2 we show how to calculate the histogram of the chosen model function and how to extract the contrast information from an experimental fringe pattern by use of this model function. In Section 3 we apply the algorithm to simulated fringe patterns. In Section 4 experimental results with real fringe patterns are obtained. Finally in Section 5 conclusions are given.

\section{Contrast Measurement from the Histogram}

The continuous histogram $h(y)$ of a continuous function $f(x)$ can be defined as the number of points $\xi$ that fulfill $y \leq f(\xi)<y+\mathrm{d} y$. The mathematical expression of this definition is

$$
h(y)=\int_{\Omega} \delta[f(x)-y] \mathrm{d} x,
$$

where $\delta$ stands for the Dirac-delta function and $\Omega$ is the set of points $x$ where we are computing the histogram. The Dirac-delta function of a continuous function $g(x)$ is ${ }^{4}$

$$
\delta[g(x)]=\sum_{i} \frac{1}{\left|g^{\prime}\right|} \delta\left(x-x_{i}\right)
$$

where $g^{\prime}$ represents derivative of $g$ with respect to $x$, $|\cdot|$ means absolute value, $x_{i}$ are the roots of $g(x)$, and $i$ is an index that runs over them. Then, if we consider

$$
g(x)=f(x)-y,
$$

by applying Eqs. (3) and (4) and taking into account that the root of Eq. (5) is $x=f^{-1}(y)$, we obtain

$$
h(y)=\int_{\Omega} \frac{1}{\left|f^{\prime}\right|} \delta\left[x-f^{-1}(y)\right] \mathrm{d} x,
$$

or

$$
h(y)=\frac{1}{\left|f^{\prime}\left[f^{-1}(y)\right]\right|} .
$$

Expression (7) can be rewritten, by means of the inverse function theorem, as

$$
h(y)=\left|\frac{\mathrm{d} f^{-1}(y)}{\mathrm{d} y}\right| .
$$

Equations (7) and (8) are a well-known result of the theory of probability if we interpret the histogram of a function as the probability distribution for the val- ues of $y .{ }^{5}$ From Eq. (6) it is clear that the conditions for $f(x)$ to have a histogram are the existence of the inverse function $f^{-1}(y)$ and the absence of extrema of $f(x)$ within $\Omega$. For these reasons, in the case of periodic functions, we cannot directly apply Eqs. (7) or (8). To compute the histograms, we must use only one semiperiod between a minimum and a maximum and multiply the histogram by the number of semiperiods present. For instance, to compute the continuous histogram of $f(x)=a+b \cos (w x), a>b$, we use the interval $\Omega=(0, \pi / w)$ where no extrema are present and $f^{-1}(y)$ is defined. Then, applying Eq. (7), we obtain

$$
h(y)=\left\{\begin{array}{cc}
1 /\left|\omega\left[b^{2}-(y-a)^{2}\right]^{1 / 2}\right| & a-b<y<b+a \\
0 & \text { elsewhere }
\end{array} .\right.
$$

In this way, when our fringe pattern corresponds to lines of any shape with sinusoidal profile, we can fit its histogram with the one given by Eq. (9), and from the fitting parameters we can obtain the contrast by $C=|b / a|$. However, if we use Ronchi rulings to determine the CTF of an optical system, this simple scheme does not work properly because of noise in the fringe pattern and discontinuities of the model, as explained in Subsections 2.A-2.D.

\section{A. Histogram of a Noisy Signal}

When a fringe pattern is obtained by means of a CCD camera or by other procedures, there always exists noise that modifies the histogram shape. We will assume an additive noise $n(\mathbf{r})$ whose probability distribution is $p(y)$, where $\mathbf{r}$ is the position vector. The histogram $h(y)$ of the fringe pattern $I(\mathbf{r})$ can be considered to be the probability distribution of the intensity values. Thus the histogram of a noisy image,

$$
\bar{I}(\mathbf{r})=I(\mathbf{r})+n(\mathbf{r}),
$$

will be the probability distribution of a signal composed by the sum of two signals with probability distributions $h(y)$ and $p(y)$. From probability theory ${ }^{5,6}$ it can be proved that the histogram of $\bar{I}(\mathbf{r})$ is

$$
\bar{h}(y)=h(y) * p(y),
$$

where $*$ denotes the convolution product. Then, by selecting a model for the additive noise, we can determine the histogram of the selected model with additive noise as the convolution product of the model histogram with the noise histogram.

\section{B. Elimination of Divergences}

Another problem of the continuous histogram [Eq. (7)] are the divergences that exist near the extrema of the model, where $f^{\prime}(x)=0$. The presence of these divergences destabilizes the minimization algorithm necessary for the fitting process, making it difficult and unreliable. We solved this problem by using a sampled model instead of a continuous one. For a sampled model with $M$ samples it is clear that the histogram will never diverge, the fitting making eas- 
ier and more reliable. This solution is not really an approximation, since any digital image acquisition system imposes a spatial sampling and an intensity quantization.

The first step to compute the discrete histogram of a sampled function is to define the range of values for the intensities and the domain of the sampled function. The possible values for the intensities are

$$
y_{n}=(n-1) \Delta y, \quad n=1, \ldots, N,
$$

where $\Delta y$ is the difference between two adjacent intensity levels and we are assuming that the lowest value for the intensity is 0 . The domain $\Omega$, where the sampled function is defined, is given by a uniform sampling,

$$
x_{m}=x_{1}+(m-1) \Delta x, \quad m=1, \ldots, M,
$$

where $x_{1}, \Delta x$, and $M$ are free parameters that determine this sampling.

The discrete histogram $h[n]$ (where the square brackets indicate that the discrete histogram can have only positive integer values) of the sampled
The +1 added to $h\left[n_{\mathrm{MAX}}\right]$ is to take into account the final point $\left[x_{M}, f\left(x_{M}\right)\right]$. From Eq. (15) it is easy to verify that

$$
\sum_{n=1}^{N} h[n]=M
$$

where $M$ is the total number of sampling points.

Then Eqs. (15) and (16) define the way a discrete histogram of a sampled function can be computed. However, working with integer parts and absolute values is not comfortable from the viewpoint of the analysis, so we are going to define a continuous histogram of a sampled model function, $h(n)$, from its discrete counterpart, $h[n]$. To eliminate the absolute values, we are going to consider only model functions $f(x)$ monotonically increasing in the range $\left[x_{1}\right.$, $\left.x_{M}\right]$. This is not a lack of generalization, because the histogram of a monotonically decreasing function is the same as the corresponding mirror reflection about the vertical axis. With this consideration and when the integer parts of Eq. (15) are eliminated, the continuous histogram of the sampled function, $h(n)$, is

$$
h(n)= \begin{cases}{\left[f ^ { - 1 } \left(y_{\left.\left.n_{\mathrm{MIN}}+\Delta y / 2\right)-x_{1}\right] / \Delta x}\right.\right.} & n=n_{\mathrm{MIN}} \\ {\left[f^{-1}\left(y_{n}+\Delta y / 2\right)-f^{-1}\left(y_{n}-\Delta y / 2\right)\right] / \Delta x} & n_{\mathrm{MIN}}<n<n_{\mathrm{MAX}} \\ {\left[x_{M}-f^{-1}\left(y_{n_{\mathrm{MAX}}}-\Delta y / 2\right)\right] / \Delta x+1} & n=n_{\mathrm{MAX}} \\ 0 & \text { elsewhere }\end{cases}
$$

function $f\left(x_{m}\right)$ is defined as the number of points $x_{m}$ such that

$$
\begin{aligned}
y_{1} & \leq f\left(x_{m}\right)<y_{1}+\Delta y / 2, & & n=1, \\
y_{n}-\Delta y / 2 & \leq f\left(x_{m}\right)<y_{n}+\Delta y / 2, & & 1<n<N, \\
y_{N}-\Delta y / 2 & \leq f\left(x_{m}\right)<y_{N}, & & n=N .
\end{aligned}
$$

With this definition $h[n]$ is basically calculated by means of counting the number of points $x_{m}$ in the interval $\left[f^{-1}\left(y_{n}-\Delta y / 2\right), f^{-1}\left(y_{n}+\Delta y / 2\right)\right]$, that is, where $n_{\mathrm{MAX}}$ and $n_{\mathrm{MIN}}$ are defined by Eq. (16). Again, from Eq. (18) it is easy to verify that

$$
\sum_{n=1}^{N} h(n)=M
$$

so no divergences appear as long as $f^{-1}\left(y_{n}\right)$ exists. Also, in the limit $\Delta x \rightarrow 0, \Delta y \rightarrow 0$, Eq. (18) is the link between the discrete histogram of a sampled function and the corresponding continuous histogram of a con-

$$
h[n]= \begin{cases}\left|\operatorname{int}\left[f^{-1}\left(y_{n_{\mathrm{MIN}}}+\Delta y / 2\right) / \Delta x\right]-\operatorname{int}\left(x_{1} / \Delta x\right)\right| & n=n_{\mathrm{MIN}} \\ \left|\operatorname{int}\left[f^{-1}\left(y_{n}+\Delta y / 2\right) / \Delta x\right]-\operatorname{int}\left[f^{-1}\left(y_{n}-\Delta y / 2\right) / \Delta x\right]\right| & n_{\mathrm{MIN}}<n<n_{\mathrm{MAX}} \\ \left|\operatorname{int}\left(x_{M} / \Delta x\right)-\operatorname{int}\left[f^{-1}\left(y_{n_{\mathrm{MAX}}}-\Delta y / 2\right) / \Delta x\right]\right|+1 & n=n_{\mathrm{MAX}} \\ 0 & \text { elsewhere }\end{cases}
$$

where $\operatorname{int}(x)$ denotes the integer part of $x$ and $n_{\mathrm{MAX}}$ and $n_{\mathrm{MIN}}$ are the first and the final indices with $h[n]$ different from 0 . They are computed by

$$
\begin{aligned}
& n_{\mathrm{MAX}}=\operatorname{int}\left[f\left(x_{M}\right) / \Delta y\right]+1, \\
& n_{\mathrm{MIN}}=\operatorname{int} f\left[\left(x_{1}\right) / \Delta y\right]+1 .
\end{aligned}
$$

tinuous function given by Eqs. (7) and (8). Thus Eq. (18) is the expression of the histogram we adopted to make the calculations. Figure 1 shows the relation between $h[n]$ and $h(n)$ for the model function $f(x)=$ $a+b \cos (w x)$ with $b=75, a=125 ; x_{1}=0, x_{M}=\pi / w$, and $M=300$.

\section{Model Function}

Once we know how to account for the effect of the noise in the histogram of the model function [Eq. 
(11)], and how to manage the divergences appearing in the continuous definition of the histogram [Eq. (18)], the next step is to select a suitable model function able to adapt to a variety of profiles, from highcontrast square patterns to almost pure sinusoidal patterns, considering a continuous grading of shapes from square to sinusoidal. The simple sinusoidal model function of Eq. (9) is not flexible enough to accomplish all this phenomenology. A good candidate for this, and the one we finally adopted, was the sigmoidal function $f_{S}(x)$ given by

$$
f_{S}(x)=a+\frac{b-a}{1+\exp \left[-\left(x-x_{0}\right) / \lambda\right]},
$$

where $a$ and $b$ are parameters that control the maximum and the minimum values of $f_{S}(x), x_{0}$ is a parameter that controls the possible lateral shift, and $\lambda$ is the parameter that controls the shape: As $\lambda$ grows, $f_{S}(x)$ changes from a step function to a sinusoidallike function. Then, as explained above for periodic signals, $f_{S}(x)$ will represent an approximation of one semiperiod. The continuous histogram of $f_{S}(x)$ is, when we apply Eq. (7) or Eq. (8),

$$
h_{S}(y)=\left\{\begin{array}{ll}
\lambda(b-a) /[(b-y)(y-a)] & y \in(a, b) \\
0 & \text { elsewhere }
\end{array} .\right.
$$

As can be seen in Eq. (21), divergences appear in $y=$ $a$ and $y=b$, making it difficult to be used as a fitting function (as explained above).

When we take into account that the inverse function of $f_{S}(x)$ is

$$
f_{S}^{-1}(y)=x_{0}-\lambda \ln \left(\frac{y-b}{a-y}\right),
$$

the continuous histogram of the sampled version of $f_{S}(x)$ is

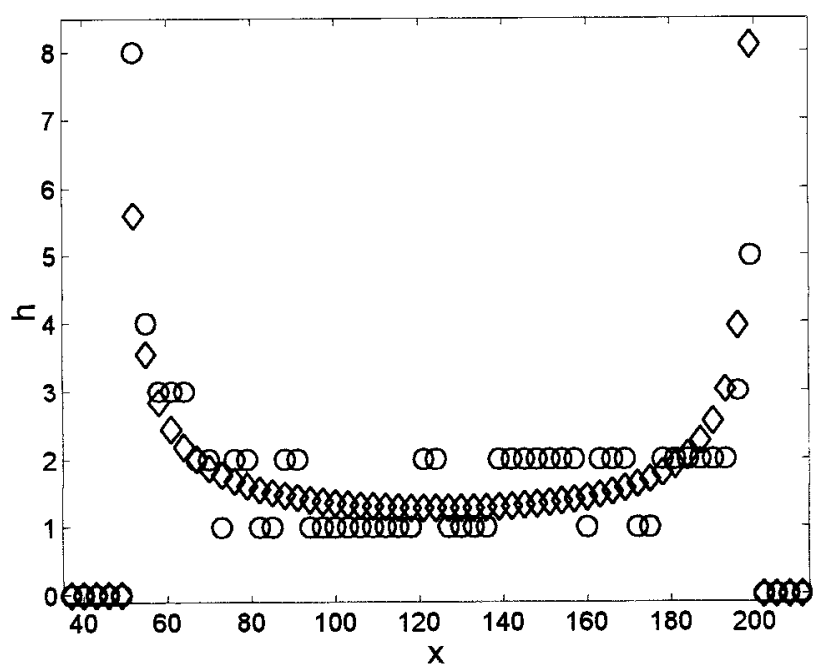

Fig. 1. Relationship between $h[n]$ (circles) and $h(n)$ (diamonds) for $f(x)=a+b \cos (w x)$, with $b=75, a=125, x_{1}=0, x_{M}=\pi / w$, and $M=300$. Only one of each of the four points is presented to improve the visibility of the figure.

where we have explicitly written the free parameters of the model and $p(n, \sigma)$ is the sampled version of Eq. (24) obtained by changing of the continuous variable $y$ with $y_{n}$ given by Eq. (12).

\section{Calculation of Contrast Parameters}

The final step in the proposed method for contrast measurement is the calculation of the parameters $a$, $b, \lambda, x_{0}$, and $\sigma$ that minimize the functional $E$ given by

$$
E=\sum_{n=1}^{N}\left\{h_{D}[n]-\bar{h}_{S}\left(n, a, b, \lambda, x_{0}, \sigma\right)\right\}^{2},
$$

where $h_{D}[n]$ is the discrete histogram of the experimental data to be analyzed, with its maximum nor-

$$
h_{S}(n)= \begin{cases}{\left[f_{\mathrm{S}}^{-1}\left(y_{n_{\mathrm{MIN}}}+\Delta y / 2\right)-x_{1}\right] / \Delta x} \\ \frac{\lambda}{\Delta x} \ln \left(\left\{1+\frac{\Delta y}{[b-(n-1 / 2) \Delta y]}\right\}\left\{1+\frac{\Delta y}{[(n-3 / 2) \Delta y-a]}\right\}\right) & n=n_{\mathrm{MIN}} \\ \left\{x_{M}-f_{S}^{-1}\left(y_{n_{\mathrm{MAX}}}-\Delta y / 2\right)\right\} / \Delta x+1 & n=n<n_{\mathrm{MAX}} \\ 0 & \text { elsewhere }\end{cases}
$$

where $n_{\mathrm{MIN}}$ and $n_{\mathrm{MAX}}$ are given by Eq. (16).

We have assumed the additive noise to be Gaussian, with zero mean, since it is easily tractable. Then its probability distribution will be

$$
p(y, \sigma)=\frac{1}{\sqrt{2 \pi \sigma}} \exp \left(-\frac{y^{2}}{2 \sigma^{2}}\right),
$$

and then the model histogram for the sigmoidal function with additive Gaussian noise incorporated in the model will be, when we apply Eq. (11),

$$
\bar{h}_{S}\left(n, a, b, \lambda, x_{0}, \sigma\right)=h_{S}(n) * p(n, \sigma),
$$

malized to 1 . From the parameters obtained in the minimization of Eq. (26) the contrast of the image with the histogram is

$$
C=\frac{f_{S}\left(x_{M}\right)-f_{S}\left(x_{1}\right)}{f_{S}\left(x_{M}\right)+f_{S}\left(x_{1}\right)} .
$$

When the noise is independent of the intensity values, Eq. (26) resumes our contrast measurement method. However, in real experiments we observed an intensity dependence on the noise level. We expect that the development of a model with multiplicative noise will solve this problem. Nonetheless, 


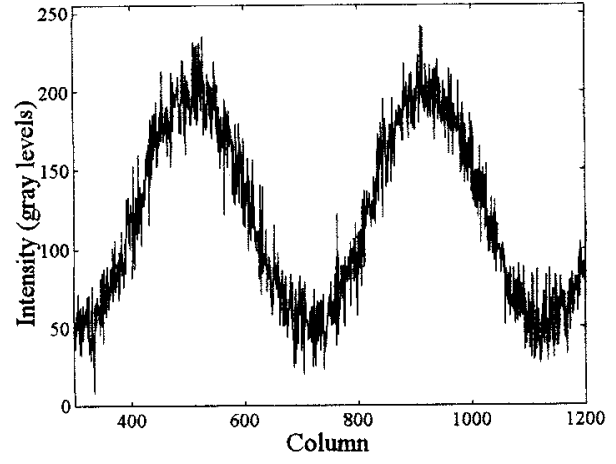

(a)

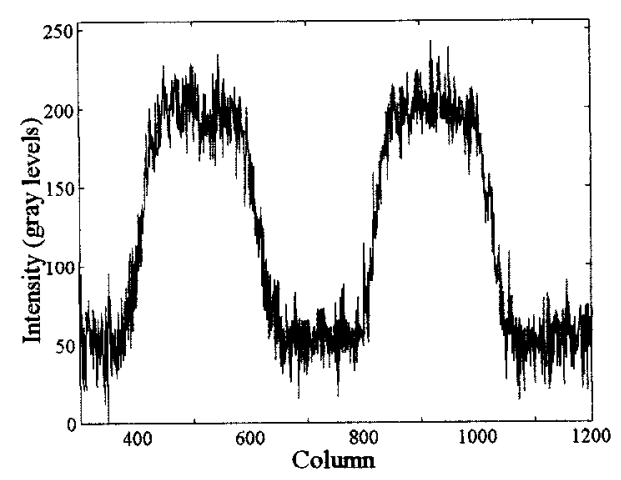

(b)

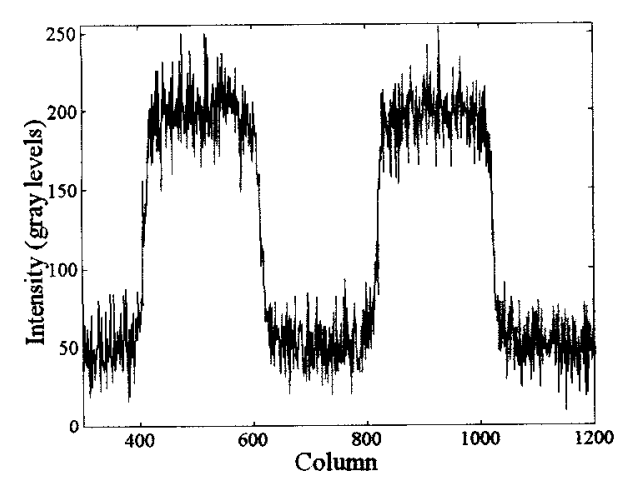

(c)

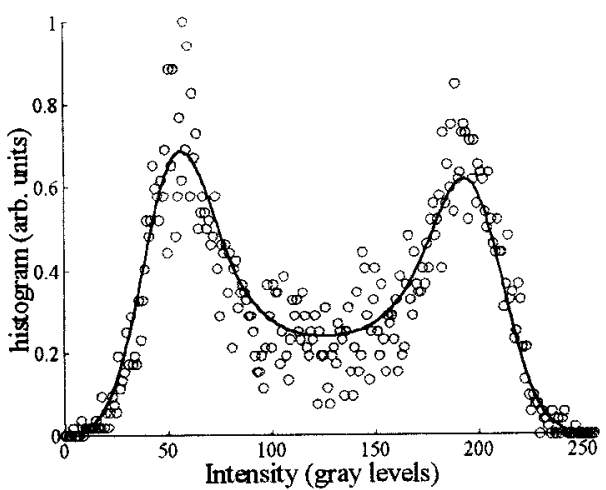

(d)

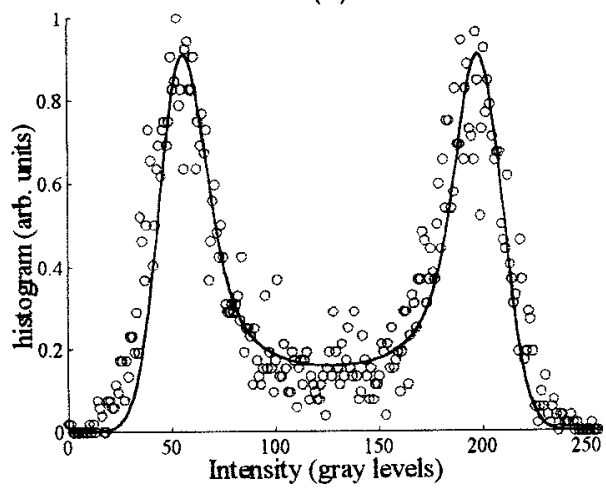

(e)

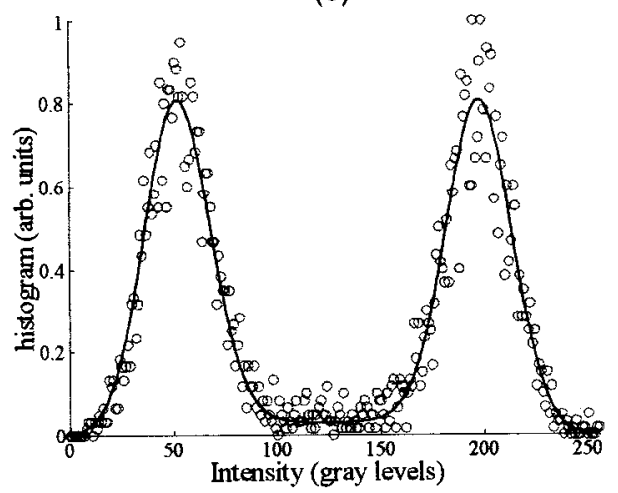

(f)

Fig. 2. Simulated one-dimensional fringe patterns with additive noise and different fringe profile: (a) for a sine fringe pattern, (b) quasi-sine fringe pattern, (c) quasi-square fringe pattern. For all cases $a=50, b=200, \sigma=15$ g.l. (d), (e), (f): circles, histograms obtained from (a), (b), (c), respectively; curve, fits to sigmoidal histogram by means of minimization of Eq. (26).

we adopted a strategy based on the additive noise model, which consists of dividing the histogram of a noisy model function into two parts and using two noise levels $\sigma_{1}$ and $\sigma_{2}$ for each one. We implemented this idea by means of a weighting function $\Lambda(n)$, defined as a step function with a linear transition zone of width $\Delta n=\operatorname{int}[\alpha(b-a)]$ gray levels and centered at $n=\operatorname{int}[(a+b) / 2]$. The parameter that controls the width of the transition zone is typically $\alpha=0.1$.

Finally, the histogram for the sigmoidal function with two levels of additive noise will be

$$
\begin{aligned}
\bar{h}_{S}\left(n, a, b, \lambda, x_{0}, \sigma_{1}, \sigma_{2}\right)= & \Lambda(n)\left[h_{s}(n) * p\left(n, \sigma_{1}\right)\right] \\
& +[1-\Lambda(n)]\left[h_{s}(n) * p\left(n, \sigma_{2}\right)\right],
\end{aligned}
$$

and the parameters are determined by the minimization of

$$
E=\sum_{n=1}^{N}\left\{h_{D}[n]-\bar{h}_{S}\left(n, a, b, \lambda, x_{0}, \sigma_{1}, \sigma_{2}\right)\right\}^{2} .
$$

From Eq. (23) it can be seen that Eqs. (26) and (29) are nonlinear minimization problems. Many algorithms exist to perform this task. In particular we used the Nelder-Mead-type simplex algorithm implemented in the optimization toolbox of the Matlab environment. ${ }^{7}$ In general the successful minimization of a nonlinear problem needs good starting values for the parameters involved. In our case the best results were obtained with the following rules for selecting the starting values. When $h_{D}[n]$ is bimodal, 


\begin{tabular}{|c|c|c|c|c|c|c|c|c|}
\hline \multirow[b]{2}{*}{ Figure } & \multicolumn{8}{|c|}{ Parameter } \\
\hline & $a^{*}$ & $b^{*}$ & $f_{S}\left(x_{1}\right)^{*}$ & $f_{S}\left(x_{M}\right)^{*}$ & $C$ & $x_{0}^{*}$ & $\lambda$ & $\sigma^{*}$ \\
\hline $2(\mathrm{a})$ & 50.67 & 203.59 & 54.01 & 200.25 & 0.575 & 0.06 & 0.263 & 14.77 \\
\hline $2(\mathrm{~b})$ & 53.07 & 204.41 & 53.36 & 204.12 & 0.586 & -0.07 & 0.160 & 12.42 \\
\hline $2(c)$ & 52.35 & 198.50 & 52.35 & 198.50 & 0.583 & 0.03 & 0.0308 & 15.67 \\
\hline
\end{tabular}

*Gray levels.

$a$ and $b$ are initialized as the gray values that correspond to each maximum of $h_{D}[n]$. If $h_{D}[n]$ presents only one lobe, whose maximum is located in the gray value $G$, the parameters $a$ and $b$ are initialized as $G-\Delta G$ and $G+\Delta G$, with $\Delta G$ typically 4 .

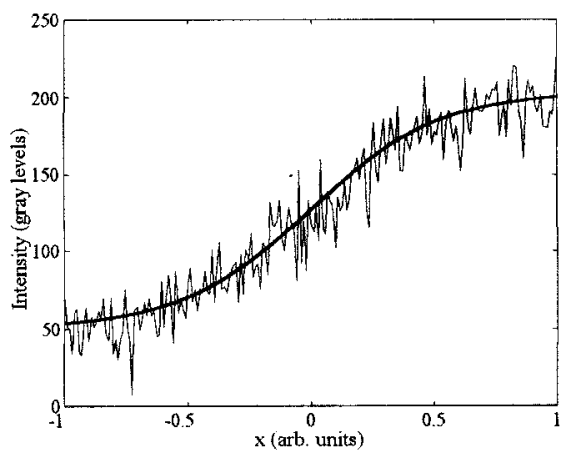

(a)

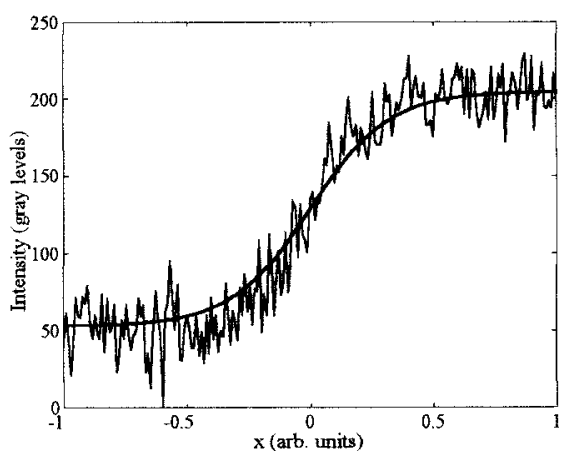

(b)

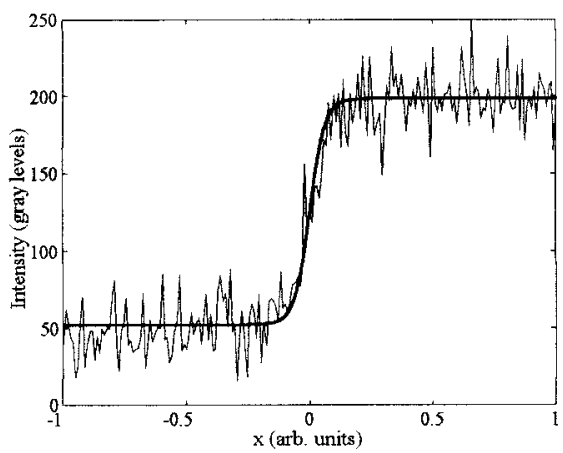

(c)

Fig. 3. Simulated (thin) and estimated (thick) fringe shape for the fringe patterns given in Fig. 1. The estimated fringe shape is obtained from results of Table 1 . As we can see, there is a good agreement between the simulated and the estimated profiles.
The shape parameter $\lambda$ is initialized as $h_{D}\left[\left(n_{a}+\right.\right.$ $\left.\left.n_{b}\right) / 2\right]$, where $n_{a}$ and $n_{b}$ are the indices of the gray values $a$ and $b$ previously initialized. If the histogram $h_{D}[n]$ is too noisy, local averaging centered on $n=\left(n_{a}+n_{b}\right) / 2$ is done for the calculation of $h_{D}\left[\left(n_{a}+\right.\right.$ $\left.\left.n_{b}\right) / 2\right]$.

Finally, the parameter $x_{0}$ is initialized to 0 , and the parameters $\sigma_{1}$ and $\sigma_{2}$ are initialized as the width at half the height of each of the lobes if the histogram is bimodal. If the histogram is monomodal $\sigma_{1}$ and $\sigma_{2}$ are initialized as the corresponding width of the unique lobe.

\section{Application to Simulated Patterns}

The algorithm depicted above was applied to three simulated fringe patterns with added noise, as shown in Fig. 2. Since we are working with 256 gray levels (g.l.) in all these cases, then $N=256, \Delta y=1$, and thus $y_{\mathrm{MIN}}=0$ and $y_{\mathrm{MAX}}=255$. We arbitrarily fixed $x_{1}=-1, x_{M}=1, \Delta x=10^{-3}, M=2001$, for all our measurements.

Figures 2(a)-2(c) show the fringe patterns that range from a sinusoidal to a square profile shape. In the three cases the actual contrast is $0.6(a=50, b=$ $200), x_{0}$ is zero, and the noise standard deviation is 15 g.l. The corresponding histograms and the result of the minimization of Eq. (26) are shown in Figs.

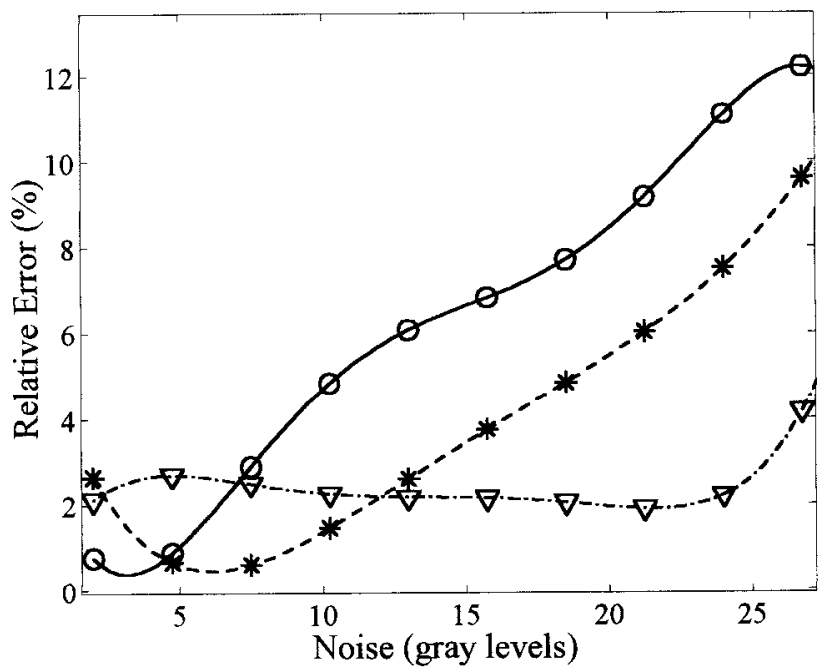

Fig. 4. Estimated uncertainty for contrast estimation in terms of Gaussian noise for several fringe shapes: (inverted triangles) $\lambda=$ 0.263 ; (asterisks) $\lambda=0.160$; (circles), $\lambda=0.0308$. In this case the contrast is $C=0.6(a=50, b=200)$. 


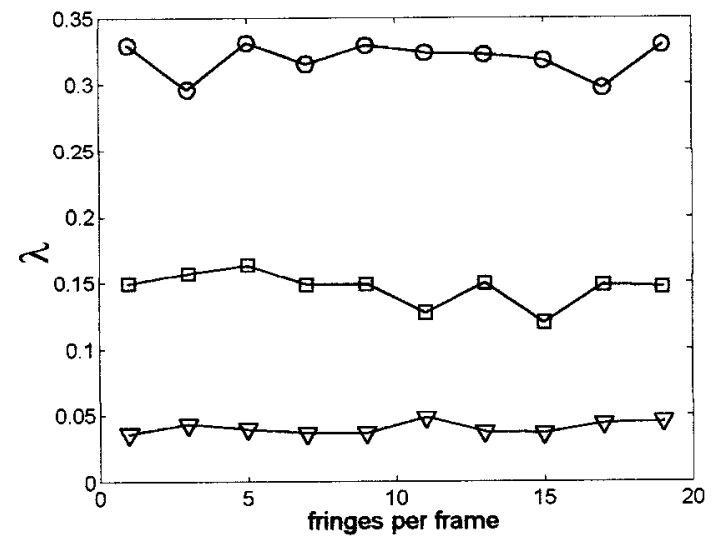

(a)

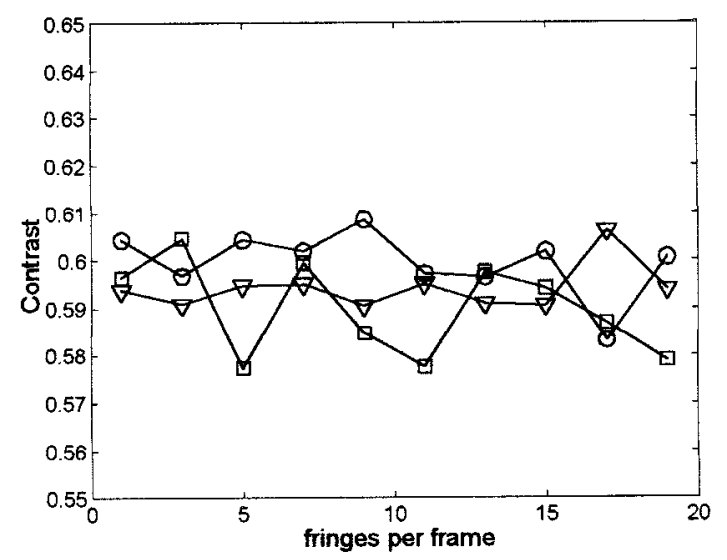

(b)

Fig. 5. (a) Estimation of $\lambda$ in terms of the number of fringes per frame for three different shape profiles, (b) estimation of contrast for the same cases. The theoretical contrast is $C=0.6$, and a noise of $10 \mathrm{~g} .1$. has been added.

2(d)-2(f). Visually the fit is good; and also numerically, as shown in Table 1.

Figure 3 represents half a period of the simulated profiles of Fig. 2 together with the sigmoidal function (20) evaluated for the corresponding parameters shown in Table 1. The profiles depicted in Figs. 3(a), $3(\mathrm{~b})$, and 3(c) correspond to the simulated profiles of Figs. 2(a), 2(b), and 2(c), respectively. In this example we can see that the sigmoidal model function adapts well from sine to square fringes.

To determine the performance of the algorithm we estimated the contrast in terms of the noise level for three different shapes: sinusoidal, quasi sinusoidal, and almost square. For each level of noise and profile shape we estimated the contrast ten times and calculated the mean relative error. In Fig. 4 the mean relative error is represented in terms of the relative error. As can be seen, the error for the square-shaped profile is less that the one corresponding to the sinusoidal and the quasi-sinusoidal ones. The reason for that behavior is that the square profile has well-defined populations that produce two well-

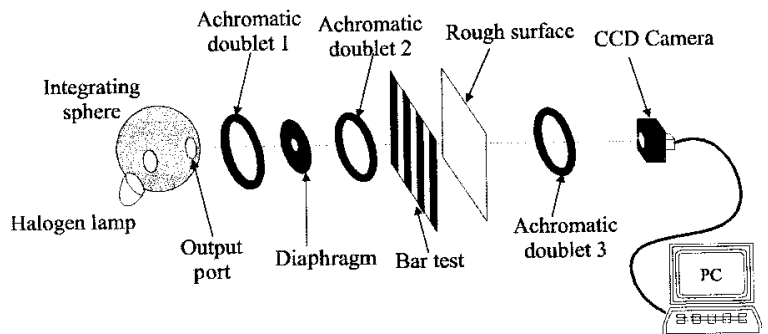

Fig. 6. Sketch of the experimental setup for contrast measurement of bar tests when a translucent rough sheet is interposed between the bar test and the CCD camera.

separated lobes in the histogram. As a consequence it is easier for the minimization algorithm to detect them correctly. In the case of the sinusoidal and the quasi-sinusoidal patterns the lobes are not so well resolved, making it more difficult to determination their positions correctly as the noise increases.

We also carried out an experiment that consists of modifying the number of fringes per frame to show that the fitting parameters do not depend on the spatial frequency of the output signal, especially the parameter $\lambda$ that accounts for the fringe shape. We obtained that the spatial frequency of the signal affects only the histogram as a scaling factor. In Fig. 5 (a) we show the parameter $\lambda$ in terms of the number of fringes per frame for three different fringe profiles. As we can see, $\lambda$ is constant for the three cases, except for a small fluctuation that is due to noise. A contrast estimation in terms of the number of fringes was also carried out [Fig. 5(b)]. For the three simulations the contrast is approximately the same.

\section{Experimental Results}

In this section we show the results of the application of the algorithm depicted above to determine the CTF of an optical system. Our particular interest was the measurement of the MTF of translucent rough screens. The first step of the method we are using is the measurement of the CTF and afterward estimation of the $\mathrm{MTF}^{1}$ from the CTF measurement.

The optical setup for the measurement of the CTF is shown in Fig. 6. The light produced by a halogen lamp is directed to the input port of an integrating sphere such that at the output port we have a nearly uniform white-light beam that is localized on a diaphragm by means of an achromatic doublet. The light emerging from the diaphragm is collimated by means of another achromatic doublet and directed to a Ronchi bar test that is projected on a rough translucent screen. The transmitted pattern is imaged on a bidimensional CCD camera by means of a third achromatic doublet. The CCD performs an 8-bit quantization (256 g.l.), and then $N=256, \Delta y=1$, $y_{\mathrm{MIN}}=0, y_{\mathrm{MAX}}=255$. For the sampling of the sigmoidal function we used $x_{1}=-1, x_{M}=1, \Delta x=$ $10^{-3}, M=2001$.

As an example, in Fig. 7 we can see the application of the algorithm to three real images. Figures 7(a)7(c) are profiles from the images recorded by the CCD 


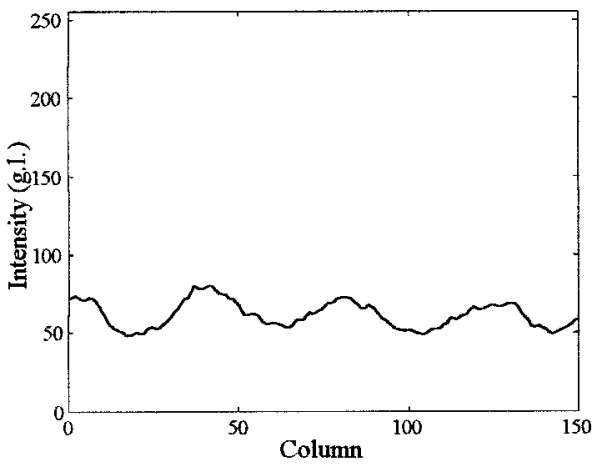

(a)

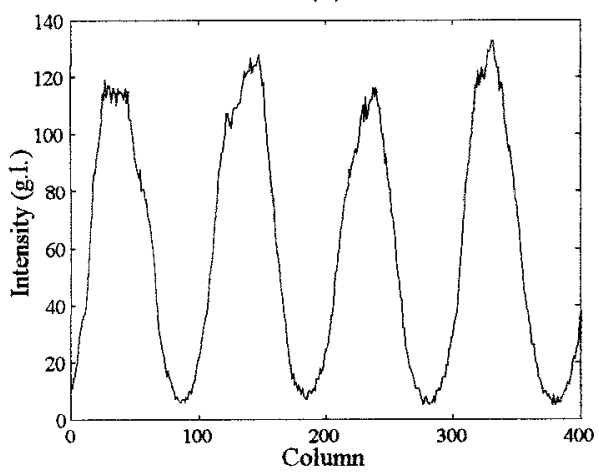

(b)

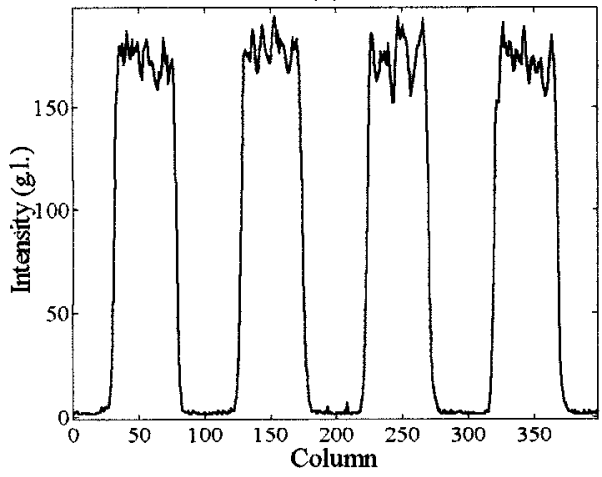

(c)

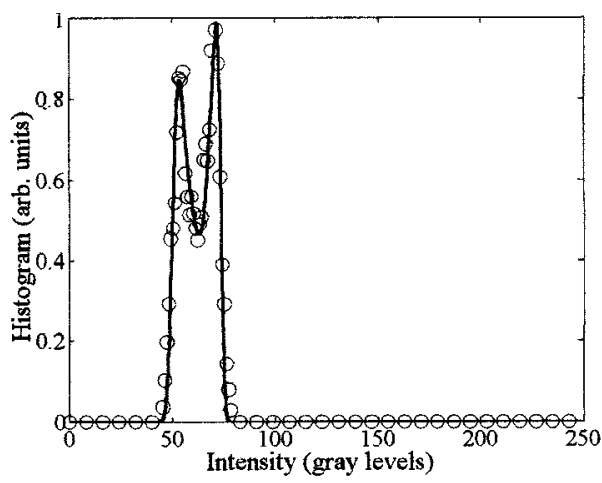

(d)

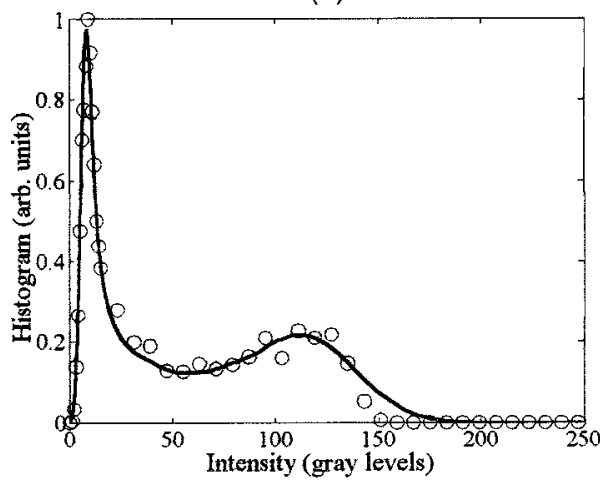

(e)

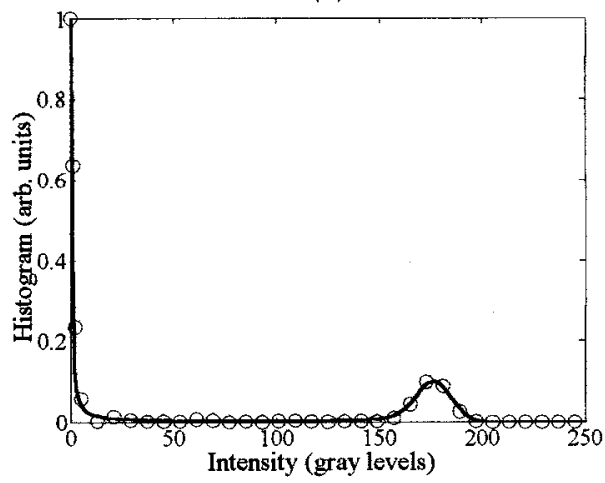

(f)

Fig. 7. Profiles from two-dimensional real fringe patterns and fits for their histograms: (a) and (d) sinusoidal low-contrast pattern, (b) and (e) sinusoidal high-contrast pattern, and (c) and (f) square high-contrast pattern. Only one of each of the four points is presented to improve the visibility of (d)-(f). Circles, experimental histogram; curve, the fit.

camera, and figures $7(\mathrm{~d})-7(\mathrm{f})$ are the experimental histograms of the corresponding images together with the results of the minimization of Eq. (29). Especially remarkable is the example of Figs. 7(a) and $7(\mathrm{~d})$; in this case the maxima of the contrast pattern are modulated, producing a lobe whose width is not due to the noise but to the intensity modulation of the maxima. Even in this case the algorithm works well and correctly locates the lobes. This shows that the parameters associated with the noise, $\sigma_{1}$ and $\sigma_{2}$, can be interpreted as high-frequency additive noise or as a low-frequency modulation present in the image, or, in general, as a mix of both effects. Table 2 shows the parameters obtained by the fits.

Finally we measured the CTF of a translucent rough sheet. The contrast of the observed pattern depends on the period of the bar test $h$, the separation between the bar test and the translucent rough sheet $d$, and its roughness $\tau / \sigma$, as shown by Garcia-Botella et al., 8 according to

$$
\begin{aligned}
\operatorname{CTF}(h, d, \sigma / \tau)= & \frac{4}{\pi} \sum_{k=0}^{\infty}(-1)^{k}\left(\frac{1}{2 k+1}\right) \\
& \times \exp \left\{-\left[(2 k+1) \frac{2 \pi}{h} \frac{\sigma d(n-1)}{\tau}\right]^{2}\right\} .
\end{aligned}
$$

In Fig. 8 we can see the fit of our experimental measurements of the CTF (obtained by the histogram-based method) to Eq. (30) in terms of spatial frequency for two values of the separation between the bar test and the sheet: $d_{1}=5 \mathrm{~cm}$ (circles) 
Table 2. Estimated Parameters for Measurements of Real Fringe Patterns by Means of a Histogram-Based Method

Parameter

\begin{tabular}{crrrrrrrr} 
& \multicolumn{1}{c}{$a^{*}$} & \multicolumn{1}{c}{$b^{*}$} & $f_{S}\left(x_{1}\right)^{*}$ & $f_{S}\left(x_{M}\right)^{*}$ & $C$ & $\lambda$ & $\sigma_{1}^{*}$ & \multicolumn{1}{c}{$\sigma_{2}{ }^{*}$} \\
\hline (a) & 47.25 & 77.25 & 50.65 & 73.85 & 0.186 & 0.486 & 2.11 & 1.26 \\
(b) & 5.64 & 122.47 & 6.61 & 121.50 & 0.897 & 0.209 & 2.25 & 24.59 \\
(c) & 0.10 & 177.35 & 0.10 & 177.35 & 0.999 & 0.0026 & 0.55 & 8.35 \\
\hline
\end{tabular}

*Gray levels.

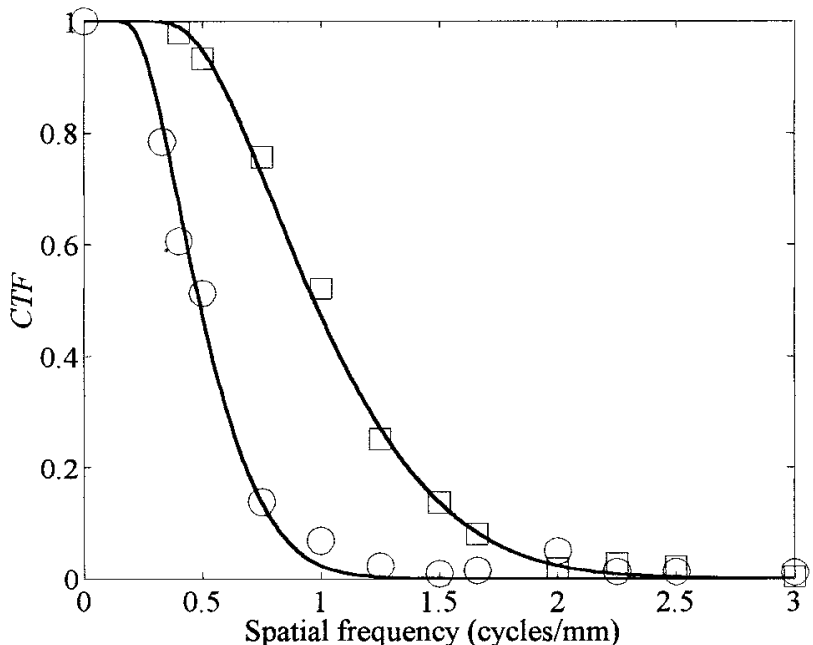

Fig. 8. Measurements of contrast transfer function by means of histogram-based method, for a dielectric rough sheet with $n=$ 1.523 and roughness parameter of $\tau / \sigma=165 \pm 4$, at distances between bar test and sheet of $d_{1}=5 \mathrm{~cm}$ (squares) and $d_{2}=10 \mathrm{~cm}$ (circles), compared with the model proposed by Garcia-Botella et $a l .8$

and $d_{2}=10 \mathrm{~cm}$ (squares). The roughness parameter for the sample was $\tau / \sigma=165 \pm 5$, measured by profilometry and reflectogoniometry. As can be seen, the agreement between the model and the experimental measurements is good. Especially remarkable is the behavior of the experimental measurements in the tails, where a small peak can be observed (Fig. 8, circles, at 2 cycles $/ \mathrm{mm}$ ). This behavior is reproducible, so we think that it corresponds to diffraction effects that the geometric model [Eq. (30)] does not consider.

\section{Conclusions}

In this study we have presented a histogram-based technique for robust contrast measurement. The method is based on the fitting of the histogram of the measured image with the histogram of a model function. Analytical expressions for the histogram of continuous as well as sampled functions were obtained. The selected model function was the sigmoidal function, which was shown to be flexible enough to accommodate a great variety of cases. With this technique contrast measurement can be performed by means of patterns of almost any shape. Finally, experimental measurements of the CTF of an optical system were made in good agreement with the theoretical model.

Juan Antonio Quiroga acknowledges the support of a postdoctoral grant of the Universidad Complutense de Madrid and the Becas Internacionales Flores-Valles program, Spain, and Centro de Investigaciones en Óptica, León, México. Luis Miguel Sanchez-Brea acknowledges the support of a predoctoral grant of the Ministerio de Educación y Cultura (Intercambio de Personal Investigador entre Industrias y Centros Públicos de Investigación) with Kinossel S.L.

This study was supported in part by Sainco Tráfico, S.A., in the framework of Proyecto de Estímulo a la Transferencia de Resultados de Investigación (PETRI) program, Baliza Luminosa para el Guiado de Tráfico Vial con Tecnología LED project of the Ministerio de Educación y Cultura.

\section{References}

1. J. W. Coltman, "The specification of imaging properties by response to a sine wave input," J. Opt. Soc. Am. 44, 468-471 (1954).

2. G. C. Holst, CCD Arrays, Cameras, and Displays (Society for Photo-Optical Instrumentation Engineers, Bellingham, Wash., 1996).

3. S. Lai and G. von Bally, "Fringe contrast evaluation by means of histograms," in OPTIKA '98: 5th Congress on Modern Optics, G. Ákos, G. Lupkovics, and P. András, eds., Proc. SPIE 3573, 384-387 (1998).

4. J. D. Gaskill, Linear Systems, Fourier Transforms, and Optics (Wiley, New York, 1978).

5. A. Papoulis, Probability, Random Variables, and Stochastic Processes (McGraw-Hill, New York, 1965).

6. B. R. Frieden, Probability, Statistical Optics, and Data Testing (Springer-Verlag, Berlin, 1983).

7. T. Coleman, M. A. Branch, and A. Grace, Optimization Toolbox for Use with MATLAB, users guide version 2 (MathWorks, Natick, Mass., 1996).

8. A. Garcia-Botella, L. M. Sanchez-Brea, D. Vazquez-Molini, and E. Bernabeu, "Modulation transfer function for translucent rough sheet," Appl. Opt. 38, 5429-5432 (1999). 